\title{
Deep sequencing uncovers commonality in small RNA profiles between transgene-induced and naturally occurring RNA silencing of chalcone synthase-A gene in petunia
}

Megumi Kasai ${ }^{1}$, Hideo Matsumura ${ }^{2}$, Kentaro Yoshida $^{3}$, Ryohei Terauchi ${ }^{3}$, Akito Taneda ${ }^{4}$ and Akira Kanazawa ${ }^{{ }^{*}}$

\begin{abstract}
Background: Introduction of a transgene that transcribes RNA homologous to an endogenous gene in the plant genome can induce silencing of both genes, a phenomenon termed cosuppression. Cosuppression was first discovered in transgenic petunia plants transformed with the CHS-A gene encoding chalcone synthase, in which nonpigmented sectors in flowers or completely white flowers are produced. Some of the flower-color patterns observed in transgenic petunias having CHS-A cosuppression resemble those in existing nontransgenic varieties. Although the mechanism by which white sectors are generated in nontransgenic petunia is known to be due to RNA silencing of the CHS-A gene as in cosuppression, whether the same trigger(s) and/or pattern of RNA degradation are involved in these phenomena has not been known. Here, we addressed this question using deep-sequencing and bioinformatic analyses of small RNAs.

Results: We analyzed short interfering RNAs (siRNAs) produced in nonpigmented sectors of petal tissues in transgenic petunia plants that have CHS-A cosuppression and a nontransgenic petunia variety Red Star, that has naturally occurring CHS-A RNA silencing. In both silencing systems, 21-nt and 22-nt siRNAs were the most and the second-most abundant size classes, respectively. CHS-A siRNA production was confined to exon 2, indicating that RNA degradation through the RNA silencing pathway occurred in this exon. Common siRNAs were detected in cosuppression and naturally occurring RNA silencing, and their ranks based on the number of siRNAs in these plants were correlated with each other. Noticeably, highly abundant siRNAs were common in these systems. Phased siRNAs were detected in multiple phases at multiple sites, and some of the ends of the regions that produced phased siRNAs were conserved.

Conclusions: The features of siRNA production found to be common to cosuppression and naturally occurring silencing of the CHS-A gene indicate mechanistic similarities between these silencing systems especially in the biosynthetic processes of siRNAs including cleavage of CHS-A transcripts and subsequent production of secondary siRNAs in exon 2. The data also suggest that these events occurred at multiple sites, which can be a feature of these silencing phenomena.
\end{abstract}

Keywords: Chalcone synthase, Cosuppression, Deep-sequencing analysis, Flower color pattern, Naturally occurring RNA silencing, Short interfering RNA

\footnotetext{
* Correspondence: kanazawa@res.agr.hokudai.ac.jp

${ }^{1}$ Research Faculty of Agriculture, Hokkaido University, Sapporo 060-8589,

Japan

Full list of author information is available at the end of the article
} 


\section{Background}

RNA silencing refers collectively to diverse RNA-mediated pathways of nucleotide-sequence-specific inhibition of gene expression. RNA silencing of genes is induced by the presence of double-stranded RNA (dsRNA) homologous to the genes. The dsRNAs are processed into small RNAs, especially 21- to 24-nulceotide (nt) short interfering RNAs (siRNAs), by a dsRNA-specific ribonuclease, Dicer or Dicer-like (DCL) proteins [1,2]. In Arabidopsis, DCL2, DCL3 and DCL4 produce 22-, 24- and 21-nt siRNAs, respectively [3]. The siRNAs are incorporated into Argonaute (AGO) proteins and serve as a guide for sequence-specific cleavage of a target RNA, leading to posttranscriptional gene silencing (PTGS) [4,5]. Transcriptional repression can also be induced by dsRNA, which contains a sequence homologous to a gene promoter and can trigger cytosine methylation on the promoter in the nuclear DNA resulting in transcriptional gene silencing (TGS) [6-8]. Like siRNAs, small RNAs called microRNAs (miRNAs) also negatively regulate the expression of endogenous genes through either RNA cleavage or the arrest of translation, which is another pathway of RNA silencing [1,9]. Small RNA (miRNA or siRNA)-mediated cleavage of an RNA can trigger the production of 21-nt secondary siRNAs either upstream or downstream of the original target site, a phenomenon called transitivity [4]. In Arabidopsis, small RNA-mediated cleavage can trigger conversion of the targeted RNA to dsRNA by RNA-dependent RNA polymerase 6 (RDR6), which is then cleaved into 21-nt phased siRNAs by DCL4. These siRNAs can include those termed transacting siRNAs (tasiRNAs), which silence other gene(s) in trans [10-12]. Small RNAs of 22 nt trigger RDR6dependent secondary siRNA production [13,14]. A recent study indicated that the presence of 22-nt RNA in either strand of the small RNA duplex is sufficient for this reaction [15].

Overexpression of the chalcone synthase- $A$ (CHS- $A$ ) gene under the control of the cauliflower mosaic virus (CaMV) 35S promoter and the nopaline synthase (NOS) terminator causes the production of white sectors or completely white flowers in transformed petunia (Petunia hybrida) plants $[16,17]$. This system was the first example of RNA silencing induced by a transgene. In these transgenic petunia plants, silencing of both the CHS-A transgene and endogenous $C H S-A$ gene was induced, so that the event was termed cosuppression [16]. The production of the wild-type pigment is inhibited because chalcone synthase performs an essential step in the biosynthesis of anthocyanins. Various silencing patterns in the petal tissues have been observed in the petunia $C H S-A$ silencing system $[18,19]$. Because it induces visibly altered phenotypes, $C H S-A$ silencing in petunia is a model system to study RNA silencing [20].
Based on the inhibition of pigmentation in flower petals, Sijen et al. demonstrated that a transgene that expresses dsRNA corresponding to the transcribed region and the promoter region induced PTGS and TGS, respectively [8]. In our recent study, we used a virus vector and succeeded in inducing heritable TGS of the endogenous $C H S$ - $A$ gene, thereby produced a plant that does not carry a transgene but has altered traits $[21,22]$.

Some of the flower-color patterns observed in transgenic petunias having cosuppression of the CHS-A genes resemble those in nontransgenic varieties [18]. One such variety is Red Star, which produces bicolor flowers having a star-type red and white pattern. As expected from the phenotypic similarity with the flowers of $C H S-A$ cosuppressed plants, the flower color pattern in Red Star was in fact demonstrated to be due to sequence-specific degradation of the $C H S$ - $A$ RNA in the white sectors [23]. Petunia breeding was launched in the 1830 s by crossing among wild species [18]. The generation of the star-type petunia flowers as a consequence of hybridization between plant lines suggests that the RNA silencing ability can be conferred via the shuffling of genomes that differ slightly from each other [20]. Similar naturally occurring RNA silencing has been reported for a picotee-type variety of petunia, which has nonpigmented sectors in the outer edge of the petal tissues [24], and for other plants such as rice [25], soybean [26-29], maize [30] and dahlia [31].

Cosuppression has been thought to be caused by a couple of mechanisms. It can be induced when multiple transgenes are integrated into the same site in the genome in an inverted orientation and fortuitous readthrough transcription over the transgenes produces dsRNA homologous to an endogenous gene in the genome, a pathway termed inverted repeat (IR)-PTGS. When sense transcripts from a transgene trigger cosuppression through RNA degradation, the pathway is referred to as sense (S)-PTGS [4]. A model for S-PTGS proposes that transgene-derived aberrant RNAs that lack a poly(A) tail or $5^{\prime}$ capping are used as a template for RDR6 to produce dsRNA, thereby triggering PTGS [4]. An alternative scenario is that nuclear-accumulated sense transcripts form imperfect hairpin structures, which resemble miRNA precursors, are processed into small RNAs and function as a trigger for RNA degradation via RDR-catalyzed synthesis of dsRNA, resulting in PTGS [32].

Our previous data indicated that $C H S-A$ cosuppression is induced by a high level of transcription of the $C H S-A$ transgene, shown by the fact that $C H S-A$ cosuppression is induced when the $C H S$ - $A$ transgene is transcribed by the CaMV $35 \mathrm{~S}$ promoter but not when the transcription from the promoter is repressed by 
epigenetic changes involving spontaneous cytosine methylation of the promoter [33]. These observations are consistent with the threshold model for induction of RNA degradation, which was first suggested on the basis of a viral RNA analysis: viral RNA degradation is triggered when the amount of viral RNA exceeds a certain level in plant cells [34]. This notion is also consistent with the fact that the frequency of cosuppression in petunia is correlated with the strength of the promoter of the $C H S-A$ transgene [35]. Thus, CHS-A cosuppression can be triggered when a particular RNA, e.g., $C H S-A$ primary transcripts or some other RNA molecule(s) derived from them, exceed a certain level. However, neither the RNA molecule(s) nor the sensing mechanism(s) of the threshold is known.

A potential trigger for $C H S$ - $A$ cosuppression in petunia has been suggested on the basis of a deep sequencing analysis of $C H S$ - $A$ siRNAs [36]. Two abundant siRNAs in antisense polarity, termed phy-siR1 and physiR2, were detected in a cosuppressed line. On the basis of the presence of these siRNAs with phased siRNAs, the authors proposed that these two siRNAs guide CHS-A mRNA cleavage and initiate the generation of phased siRNAs, leading to cosuppression. On the other hand, $C H S-A$ siRNA profiles in another cosuppressed transgenic line having inverted repeat T-DNA [37] or a petunia variety that produces picotee-type flowers [24] indicated the presence of multiple abundant siRNAs. At present, whether the population of siRNAs detected in one $C H S-A$ cosuppressed line is common to different $C H S-A$ cosuppressed lines or $C H S$ - $A$ naturally silenced lines is not known. Moreover, no insight into a general mechanism(s) of cosuppression in terms of siRNA production has been presented in any plant species.

To address these questions, here we analyzed $C H S-A$ siRNA populations from silenced and nonsilenced tissues of a transgenic line having $C H S-A$ cosuppression and a non-transgenic variety Red Star in detail. We show that multiple abundant siRNAs from $C H S$ - $A$ exon 2 are produced in the silenced tissues in both silenced lines. We also found profound commonality in siRNA production in the silenced tissues of the cosuppressed line and Red Star, which suggests the presence of a common mechanism of RNA degradation that likely depends on an evolutionary conserved feature in exon 2 of the CHS-A gene.

\section{Results}

We analyzed the mRNA and siRNAs of the CHS-A gene in the white and pigmented portions of petal tissues of petunia plants that have cosuppression or naturally occurring RNA silencing of the $C H S-A$ gene. The $C H S-A$ cosuppressed line contains a single copy of the CHS-A transgene and produces petals with a white and purple pattern. The size of the white portions is variable, but they are invariably centered on the junctions between petals; hence, the pattern is called the junction pattern [19] (Figure 1a, left). The bicolor petals of nontransgenic variety Red Star have a star-type white and red pattern: the white sector forms along the veins in the center of each petal (Figure 1a, right). In the white petal tissues of both the junction-type (J-type) and Red Star plants, $C H S-A$ mRNA was barely detected (Figure 1b) but $C H S-A$ siRNAs accumulated (Figure 1c), confirming the occurrence of CHS-A RNA degradation [23].

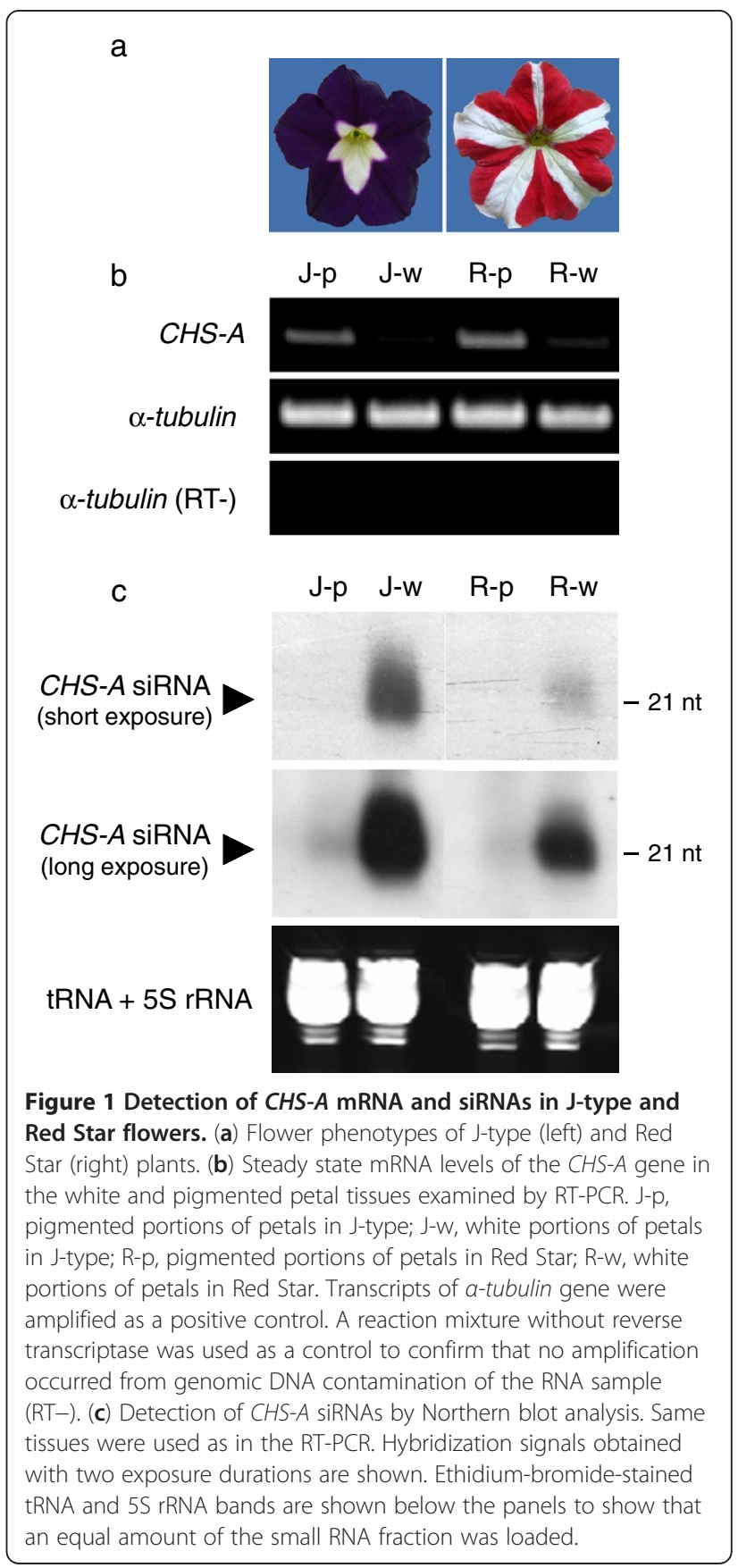


Table 1 Number of siRNA reads mapped in the CHS-A gene region

\begin{tabular}{lllll}
\hline Read statistic & J-w & J-p & R-w & R-p \\
\hline Total reads analyzed & $21,138,355$ & $26,817,315$ & $21,318,490$ & $25,612,671$ \\
\hline $\begin{array}{l}\text { Total reads mapped in } \\
\text { CHS-A region }\end{array}$ & & & & \\
\hline Sense strand & 78,684 & 3,672 & 33,958 & 2,716 \\
\hline Antisense strand & 182,513 & 7,058 & 49,627 & 1,812 \\
\hline Total & 261,197 & 10,730 & 83,585 & 4,528 \\
\hline
\end{tabular}

Abbreviations: J-p, pigmented portions of J-type petals; J-w, white portions of J-type petals; R-p, pigmented portions of Red Star petals; R-w, white portions of Red Star petals.

\section{Mapping of siRNAs on the CHS- $A$ gene in a $C H S-A$ cosuppressed line}

We analyzed siRNAs in J-type plants by deep sequencing technology. Of 21,138,355 reads, 261,197 reads matched the $C H S$ - $A$ gene region in the white portions of petals in J-type plants (Table 1). The size distribution of siRNAs mapped in the $C H S-A$ gene region revealed the predominance of siRNAs of $21 \mathrm{nt}$ and $22 \mathrm{nt}$, especially $21 \mathrm{nt}$, for both sense and antisense strands in this plant line (Figure 2a). This result indicates that $C H S-A$ siRNAs are predominantly produced by the function of DCL4 orthologue(s). The position and abundance of the 21-nt to 24-nt siRNAs mapped in the $C H S$ - $A$ gene region are shown in Figure 3. Almost all the siRNAs were mapped to exon 2 of the $C H S$ - $A$ gene region (see, for example, Figure $3 \mathrm{a}, \mathrm{b})$. There is uneven distribution of siRNA within exon 2, indicating the presence of multiple hot spots for siRNA production. CHS-A siRNAs were also detected in the purple portions of petals in J-type plants, although the level of siRNAs was $1 / 30$ of the level in the white tissues (Figure 2a, b). The presence of siRNAs at a low level in the pigmented petal tissues was also shown by the Northern blot analysis (Figure 1c, see "long exposure").

The endogenous $C H S-A$ gene and $C H S-A$ transgene have different nucleotide sequences in the $3^{\prime}$ untranslated region. siRNAs specific to the endogenous $C H S-A$ gene and those specific to the $C H S-A$ transgene were both detected (Figure 4), which indicates that mRNAs derived from the endogenous $C H S-A$ gene and $C H S-A$ transgene are both degraded via RNA silencing pathways. The number of siRNA mapped in this region was higher for the $C H S$ - $A$ transgene than for the endogenous $C H S$ - $A$ gene (Figure 4).

\section{Mapping of siRNAs on the CHS-A gene in a non- transgenic variety}

The production of $C H S$ - $A$ siRNAs was also analyzed in petal tissues of Red Star. In the white portions of petals,
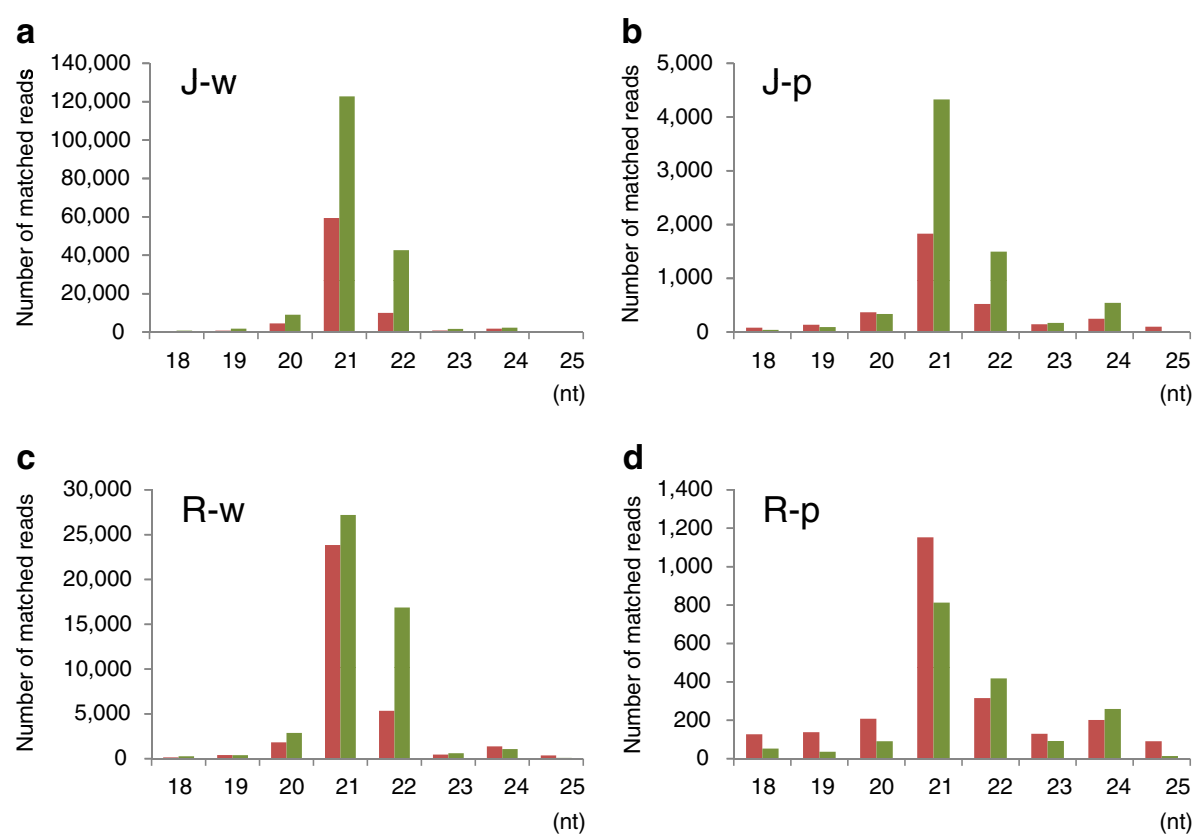

sense strand antisense strand

Figure 2 Frequency of siRNAs between 18 and $25 \mathrm{nt}$ mapped in the CHS-A gene from floral tissues of petunia. (a) White and (b) pigmented tissues of J-type plants, (c) white and (d) pigmented tissues of Red Star plants. Number of siRNAs mapped on the sense strand (red bars) and antisense strand (green bars) are indicated. 


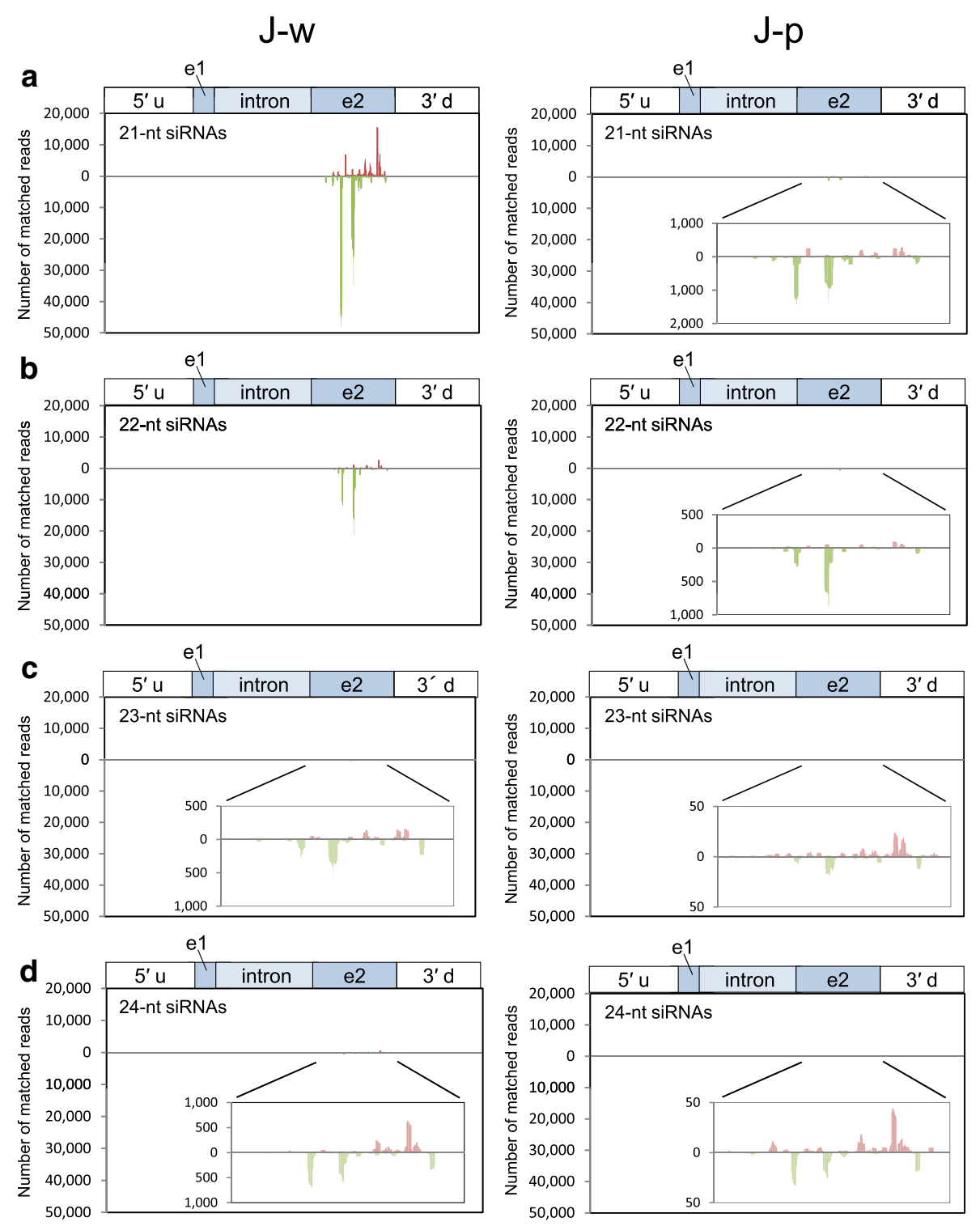

Figure 3 Position and abundance of siRNAs mapped on the CHS-A gene region in J-type plants. Data for (a) 21-nt, (b) 22-nt, (c) 23-nt and (d) 24-nt siRNAs in the white (J-w) and pigmented (J-p) petal tissues are shown. Bars above and below the $x$-axis indicate siRNAs mapped on the sense and antisense strands, respectively. A detail of the siRNA data in exon 2 is inserted when the level was very low. $5^{\prime} u, 5^{\prime}$ upstream region; e1, exon 1; e2, exon 2; 3' d, 3' downstream region.

21-nt and 22-nt siRNAs were predominant (Figure 2c). The production of siRNAs was confined to exon 2 , which included multiple highly abundant siRNAs (Figure 5), as observed for the white portions of J-type plants. Similarly, $C H S-A$ siRNAs were also detected in red portions of Red Star flowers at a very low level (1/20-1/40 of the level in white tissues; Figure 2c, d). The read number indicated that more $C H S-A$ siRNAs were detected in the white petal tissues of J-type plants than in those of Red Star plants (Table 1; Figure 2a, c). These results are consistent with the difference in the signal intensity in the Northern blot analysis (Figure 1c).
The presence of siRNAs mapped in the vicinity of the intron-exon 2 boundary

Because the distribution of $C H S-A$ siRNAs was confined to exon 2 in both J-type and Red Star plants, we had a close look at the mapping of siRNAs in the boundary between intron and exon 2. The 21-nt siRNAs mapped closest to intron in exon 2 were 13 nt and 11 nt distant from the intron-exon 2 boundary in the white portions of J-type plants for sense and antisense strands, respectively (Figure 6). Similarly, the siRNA mapped closest to the boundary was $51 \mathrm{nt}$ (data not shown) and $33 \mathrm{nt}$ (Figure 6) distant from the boundary in the white 
portions of Red Star for sense and antisense strands, respectively. In addition, 22-nt siRNAs of both sense and antisense strands were mapped at similar positions (22 nt and 9 nt distant from the boundary, respectively) in the J-type (Figure 6). Thus, the $5^{\prime}$ end of siRNA production in exon 2 was very close to the intron-exon 2 boundary in both J-type and Red Star plants.

\section{Commonality in the abundance of siRNAs between J-type and Red Star plants}

We compared the read number of 21-nt siRNAs between J-type and Red Star plants. We found that siRNAs highly abundant in the white portions of J-type plants were also highly abundant in the white portions in Red Star, and vice versa. For example, 18 of the 20 most abundant siRNAs of the sense strand (in 682 siRNA species) detected in the white portions of J-type plants were found within the 24 most abundant siRNAs (in 469 siRNA species) detected in the white portions of Red Star flowers (Figure 7a). Similarly, 16 of the 20 most abundant siRNAs of the antisense strand (in 670 siRNA species) detected in the white portions of J-type plants were found within the 23 most abundant siRNAs (in 451 siRNA species) in the white portions of Red Star plants (Figure 7b). Most strikingly, the same siRNA of antisense strand was most abundant in both J-type and Red Star plants (Figure 7b).

To compare the overall feature of siRNA production between J-type and Red Star plants, we analyzed the correlation in the rank of siRNAs based on the number of reads between J-type and Red Star plants. We calculated Spearman's rank correlation coefficient using siRNAs that had more than five reads, which cover 9799\% of all siRNA reads (see "value B / value A" in Table 2); 214 sense and 180 antisense different siRNA species, which were detected in both J-type and Red Star plants, were used for the calculation. The results indicated that the siRNA ranks in J-type and Red Star plants are highly correlated with each other for both sense and antisense strands (for the sense strand, $r_{s}=0.723, P<0.01$; for the antisense strand, $r_{s}=0.852$, $P<0.01$ ) (Table 2). Taken together, these results indicate commonality between J-type and Red Star plants in terms of siRNA production.

A similar correlation in the rank of siRNAs between J-type and Red Star plants was also detected for 22-nt siRNAs (Additional file 1: Figure S1). For example, the two most abundant siRNAs were common to J-type and Red Star plants for both sense and antisense strands. 


\section{R-w}
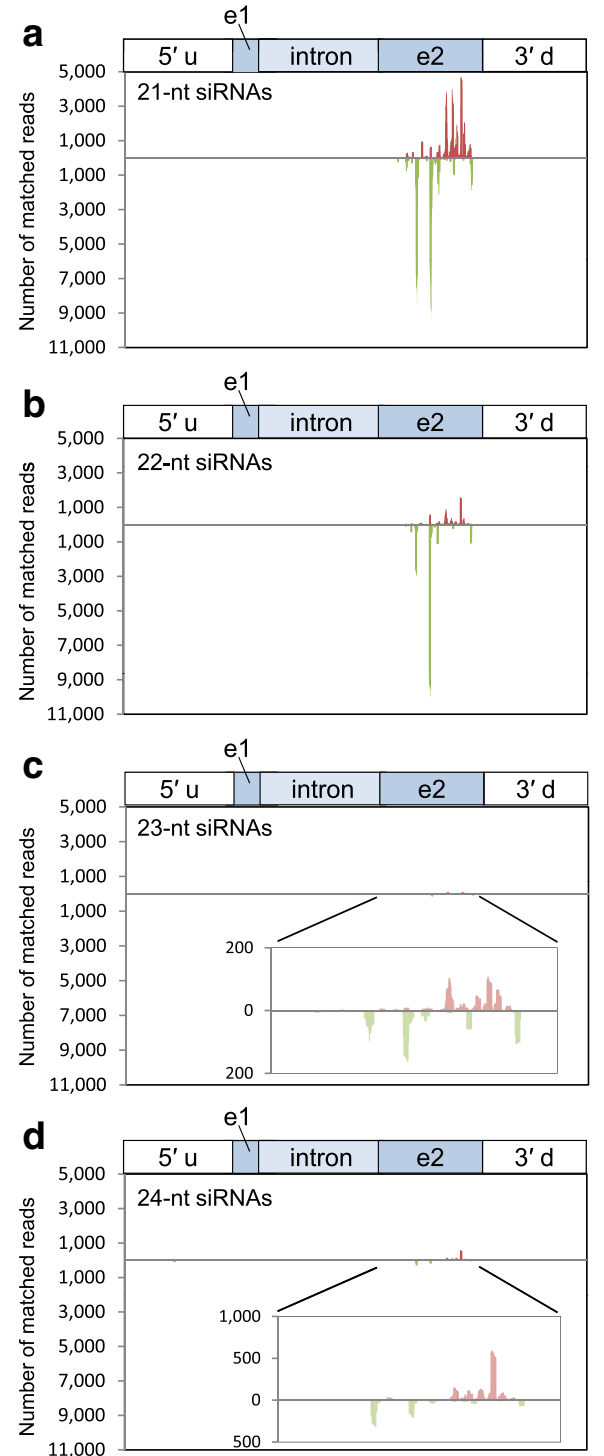
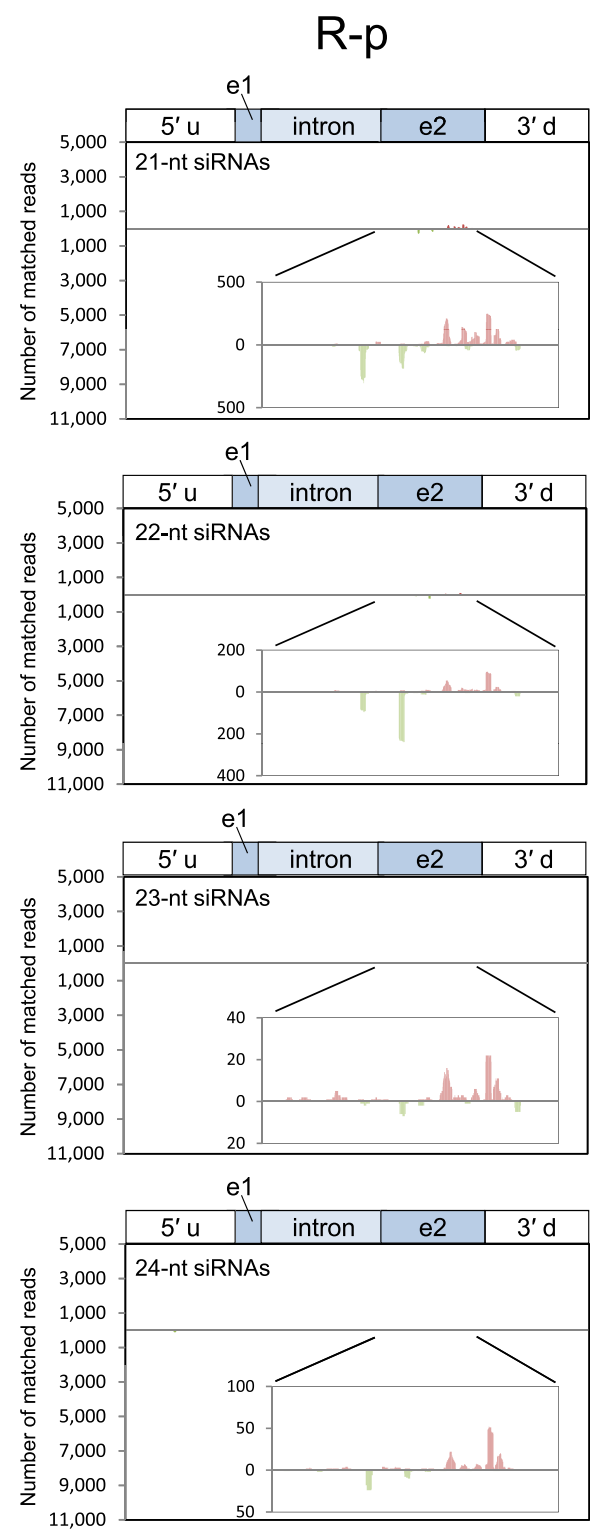

Figure 5 Position and abundance of siRNA mapped on the CHS-A gene region in Red Star plants. For more details, see Figure 3 legend.

\section{Commonality in the production of phased siRNAs}

In Arabidopsis, cleavage of transcripts by a small RNA can result in in-phase generation of 21-nt secondary siRNAs by DCL4 after production of dsRNA by RDR6 $[11,12]$. To detect phased siRNAs in the J-type and Red Star plants, we mapped siRNAs of the CHS-A gene independently in 21 different phases. Figures 8 and 9 show the distribution of 21-nt phased siRNAs that are contiguous for three or more units in each phase in exon 2. These phased siRNAs were detected in all 21 phases in both J-type and Red Star plants for both sense (Figure 8) and antisense (Figure 9) strands except for "phase 2" of the antisense strand in Red Star (Figure 9).
Phased siRNAs were distributed more widely in J-type plants than in Red Star plants: the 5' end of phased siRNA-producing region in J-type was 151-nt and 254-nt upstream of that in Red Star for sense and antisense strands, respectively, while the $3^{\prime}$ end of phased siRNA-producing region encompassed the 3 ' end of the $C H S$ - $A$ coding region in both J-type and Red Star plants. Both siRNA reads and phasing scores were consistent with a wider distribution of phased siRNAs in J-type than in Red Star (Figures 10 and 11). The maximum number of contiguous units was 19, which covers a 399-nt region (in J-type antisense strand, phase 10) (Figure 9). Some of the $5^{\prime}$ ends or 3 ' ends of the regions that produced phased siRNAs in Red Star 


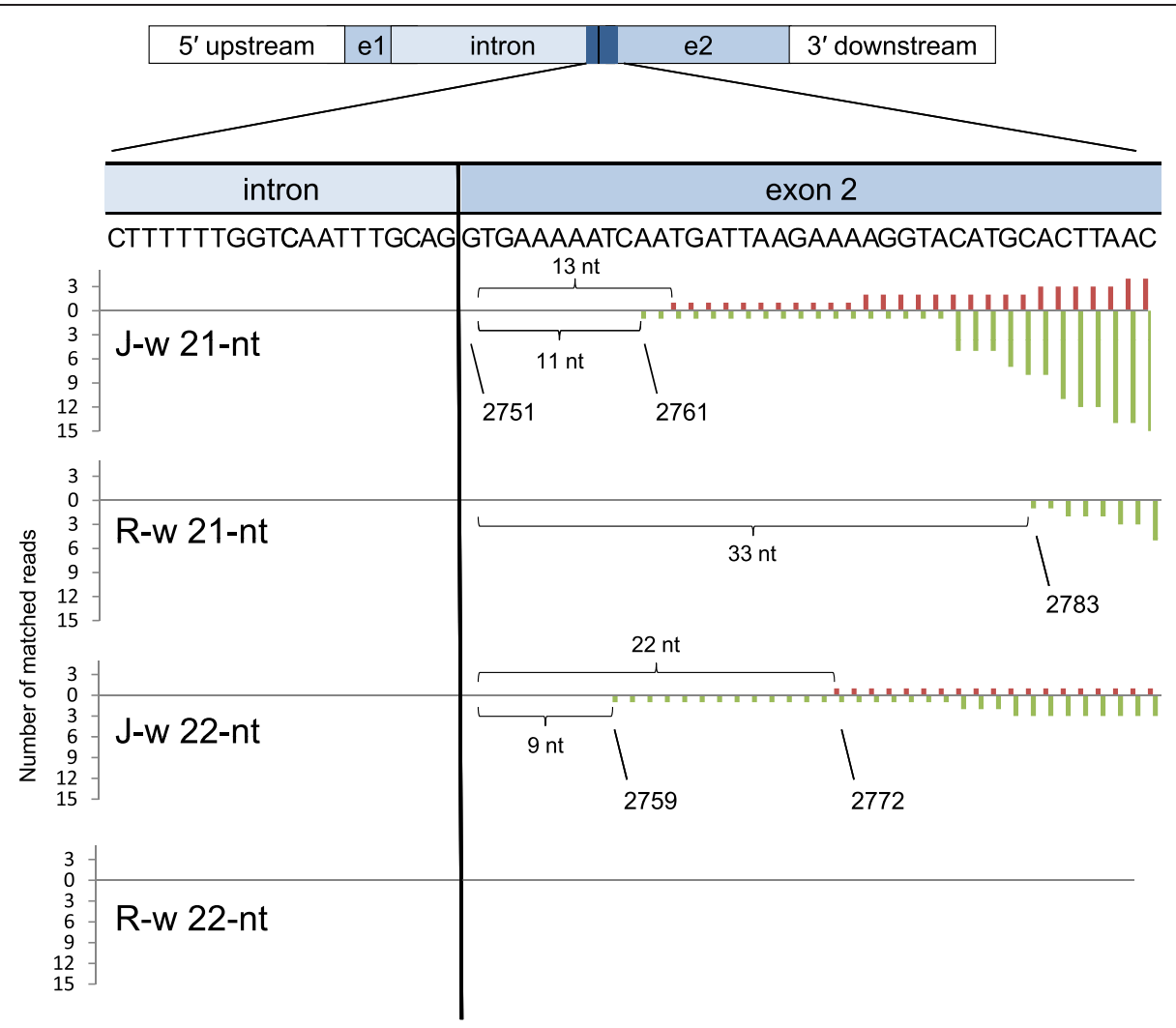

Figure 6 Position and abundance of siRNAs mapped in the boundary region between intron and exon2 of the endogenous CHS-A gene. Data for 21-nt and 22-nt siRNAs in the white petal tissues of J-type (J-w) and Red Star (R-w) plants are shown. DNA sequence in the intron-exon 2 boundary (20 nt of intron and $40 \mathrm{nt}$ of exon 2; nucleotide positions 2731-2790) is shown. Bars above and below the $x$-axis indicate siRNAs mapped on the sense and antisense strands, respectively. Nucleotide positions of the $5^{\prime}$ end of exon 2, and those of the 3' ends of antisense siRNAs mapped in this region are indicated. No 22-nt siRNA was mapped in this region in R-w.

plants were conserved in J-type plants; of the 62 regions that produced phased siRNAs for sense strand in Red Star plants (Figure 8, indicated by blue lines), 21 of the $5^{\prime}$ ends and 16 of the $3^{\prime}$ ends were conserved in J-type plants (Figure 8, indicated by red dots). Similarly, of the 50 regions that produced phased siRNAs for antisense strand in Red Star plants, 19 of the $5^{\prime}$ ends and 12 of the 3 ' ends were conserved in J-type plants (Figure 9). We also found that 21-nt siRNAs mapped in the vicinity of intron-exon 2 boundary in the antisense strand in J-type plants (Figure 6) were phased siRNAs (Figure 9; phase 10).

Overall, these data indicate that phased siRNAs were produced in multiple phases at multiple sites over exon 2 in both J-type and Red Star plants. The presence of common ends of phased siRNAs suggests that the mechanism(s) of the production of phased siRNAs, including the sites of RNA cleavage to initiate phased siRNA production, is considerably conserved between these plants for both sense and antisense strands.

\section{Discussion}

Small RNA profiles suggest a common mechanism of RNA degradation in cosuppression and naturally occurring RNA silencing of the CHS-A gene

We found that various features of small RNA production in white petal tissues are common to J-type and Red Star plants: predominant size class, exon-2-specific production, the highly abundant species, and in-phase production of siRNAs. Multiple abundant 21-nt or 22-nt siRNAs can be produced from DCL cleavage of secondary-structured nascent $C H S-A$ transcripts. They may cleave $C H S-A$ RNA with AGO orthologue(s) to trigger secondary siRNA production. Alternatively, these abundant siRNAs can be a product of DCL cleavage of dsRNAs synthesized by an RDR6 orthologue(s) from the nascent transcripts or AGO-cleaved transcripts (Additional file 2: Figure S2). It is also possible that the dsRNAs are formed by intermolecular RNA interaction [38]. In these scenarios, differences in the abundance of siRNAs reflect differences in the efficiency 


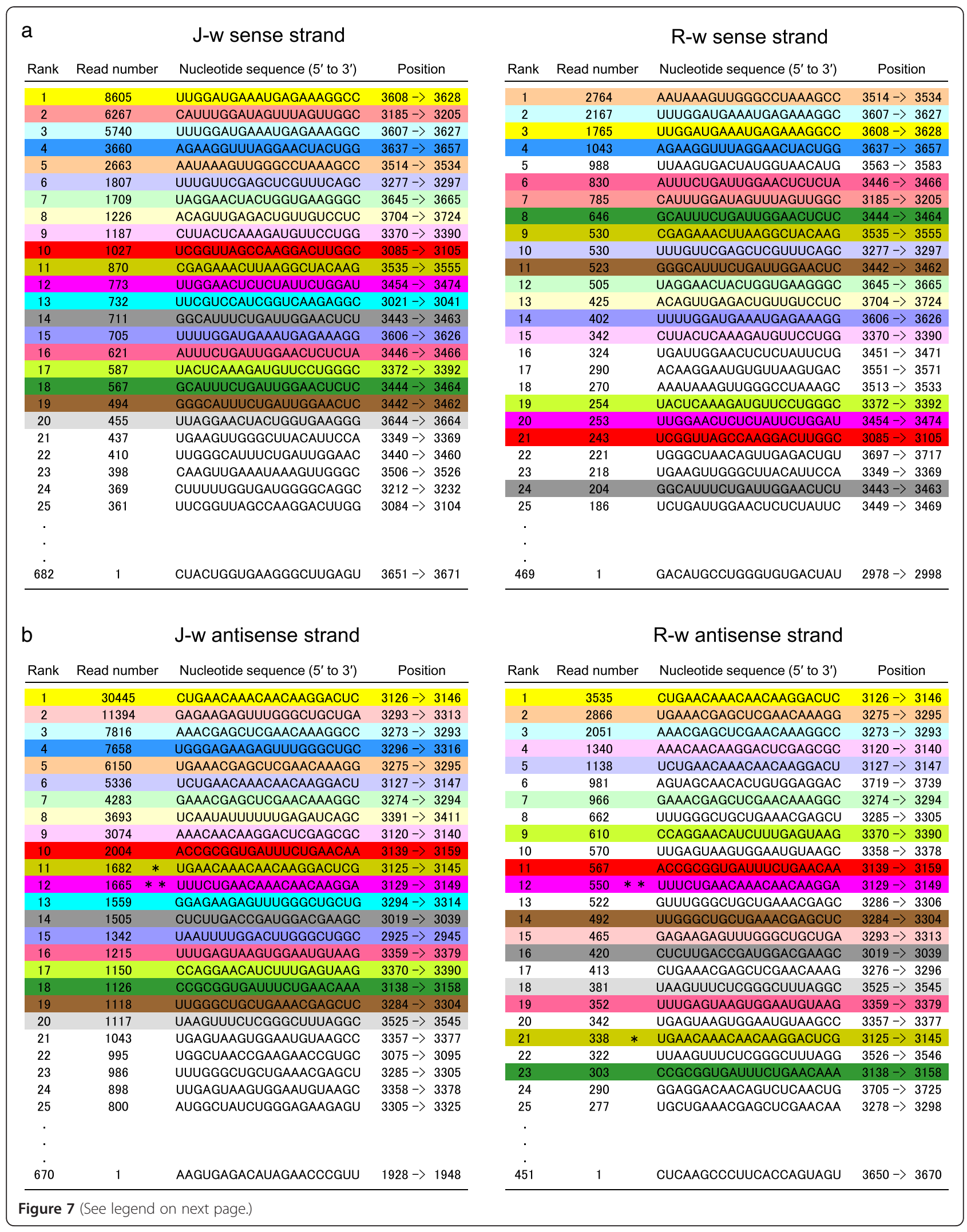


(See figure on previous page.)

Figure 7 Highly abundant 21-nt siRNAs in the white portions of petals in J-type and Red Star plants. The order of siRNAs of sense (a) and antisense (b) strands was arranged according to the number of reads in deep-sequencing analysis. J-W and R-w refer to the siRNAs detected in the white portions of J-type and Red Star plants, respectively. Twenty most abundant siRNAs in J-w are colored, and the siRNAs of the same sequence in Red Star are colored the same. Note that most of the highly abundant siRNAs in J-type plants are also highly abundant in Red Star plants. Phy-siR1 and phy-siR2 [36] are indicated by single and double asterisks, respectively.

of these biosynthetic processes or in the stability of siRNAs possibly mediated by association with AGO orthologue(s). The presence of common siRNAs suggests that sequence and/or structural preference in these processes is highly conserved in the two silencing systems.

Phased siRNAs of multiple phases were detected in this study. The presence of common ends of the regions that produced the phased siRNAs between J-type and Red Star plants suggests that the positions of the cleavages of $C H S-A$ transcripts and subsequent production of secondary siRNAs are conserved. In addition, the mapping data suggested that phased siRNAs were produced from neighboring phases, the $5^{\prime}$ or $3^{\prime}$ end of which was mapped at positions that differed by one nucleotide (Figure 12). A mechanism that could allow this phenomenon is the production of siRNAs of more than one phase by a single cleavage, but no evidence for this scenario has been reported. Alternatively, the primary siRNAs that determine the initiation site of phasing might be produced from positions differed by one or a few nucleotides. The fact that highly abundant siRNAs were mapped at positions that are very close to each other (see below) is consistent with the notion that the primary siRNAs may be produced from such a limited place. Because of the presence of phased siRNAs of various phases at various regions of exon 2 , we propose that, irrespective of the pathway of initial production of dsRNA, RNA cleavage at various sites that initiate production of secondary siRNAs can be a feature of both

Table 2 Reads and rank correlation of 21-nt siRNAs in white tissues of J-type and Red Star petals

\begin{tabular}{|c|c|c|c|c|}
\hline \multirow[t]{2}{*}{ Read statistic } & \multicolumn{2}{|c|}{ Sense strand } & \multicolumn{2}{|c|}{$\begin{array}{l}\text { Antisense } \\
\text { strand }\end{array}$} \\
\hline & J-w & R-w & $J-w$ & R-w \\
\hline Total number of siRNA reads (value A) & 59,386 & 23,840 & 122,753 & 27,197 \\
\hline $\begin{array}{l}\text { Total number of reads for siRNA } \\
\text { species with }>5 \text { reads (value B) }\end{array}$ & 58,705 & 23,287 & 122,033 & 26,646 \\
\hline Value B / value A & 0.989 & 0.977 & 0.994 & 0.980 \\
\hline Total number of siRNA species & 682 & 469 & 670 & 451 \\
\hline $\begin{array}{l}\text { Number of siRNA species with }>5 \\
\text { reads }\end{array}$ & 374 & 219 & 337 & 182 \\
\hline $\begin{array}{l}\text { Number of siRNA species with }>5 \\
\text { reads in both J-w and R-w }\end{array}$ & 214 & & 180 & \\
\hline Rank correlation coefficient $\left(r_{s}\right)$ & $0.723^{\mathrm{a}}$ & & $0.852^{\mathrm{a}}$ & \\
\hline
\end{tabular}

a $P<0.01$.

Abbreviations: J-w, white portions of J-type petals; R-w, white portions of Red Star petals. cosuppression and naturally occurring RNA silencing of the CHS-A gene (Additional file 2: Figure S2).

\section{Exon-2-specific production of siRNAs}

In both J-type and Red Star plants, siRNA production was almost always confined to exon 2. Moreover, the 5' end of siRNA production in exon 2 was very close to intron. These observations suggest that the primary event of $C H S$ - $A$ RNA degradation occurred in exon 2, and subsequent transitive RNA degradation did not reach the intron across the intron-exon 2 boundary.

It is possible that this phenomenon is associated with splicing. In fact, the presence of intron and/or splicing can suppress RNA silencing in plants $[39,40]$. In this regard, binding of factors involved in splicing, e.g., U2 auxiliary factors that bind to the 3 ' splice site upon splicing [41] or splicing factors that remain associated with the exon-exon junction even after splicing is completed [42], might inhibit progression of dsRNA synthesis over the intron-exon boundary. However, in the white tissues of J-type plants not only the endogenous $C H S$ - $A$ gene transcripts but also the $C H S-A$ transgene transcripts were degraded, while very few siRNAs were produced outside exon 2 . These observations indicate that exon 2-specific production of siRNAs occurred even on transcripts lacking an intron. Therefore, there may be mechanism by which siRNA production from CHS $-A$ transgene transcripts may be affected in trans, if splicing or spliceosome formation is involved in the exon-2-specific production of $C H S$ - $A$ siRNAs.

An alternative model to explain the exon-2-specific siRNA production is that the $5^{\prime}$ end of RNA degradation can be determined by an siRNA that targets a position in the vicinity of the intron-exon 2 boundary. The "two-hit trigger" model suggests that transitivity occurs in an RNA segment between two positions that are targeted by small RNAs [43]. According to this model, the observed siRNA production can be explained by the presence of siRNA that targets exon 2 in the vicinity of the intron-exon 2 boundary and another siRNA that targets a position downstream. Candidate siRNAs that may terminate degradation are those mapped in the vicinity of intron-exon 2 boundary (Figure 6).

Production of siRNAs that is essentially confined to exon 2 has also been observed for naturally occurring silencing of the CHS genes in soybean $[28,44]$ and dahlia [31]. These results, together with the observations 


\section{Sense strand}

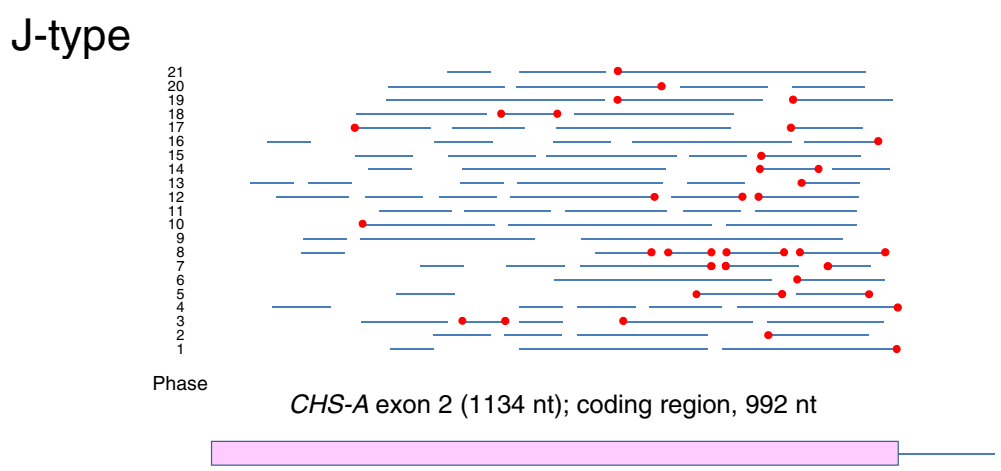

Red Star
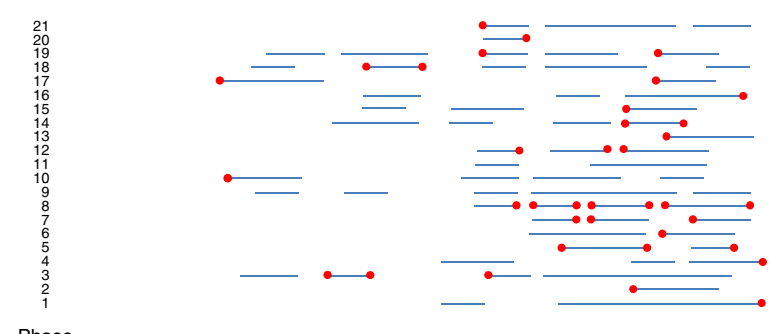

3 units of phased siRNAs (63 nt)

Figure 8 Phased siRNAs from sense strand of CHS-A exon 2. Data in upper and lower panels were obtained from white portions of J-type and Red Star petals, respectively. Presence/absence of 21-nt siRNAs was analyzed in 21 phases independently. The results of each phase are marked 1-21: the first nucleotide of "phase 1" corresponds to the first and last nucleotides of the CHS-A reference sequence (see Methods) for sense and antisense strands, respectively. Blue lines: regions producing phased siRNAs of three or more contiguous units. Red dots: 5' or 3' ends of phased-siRNA producing regions that are common to J-type and Red Star petals.

regarding the petunia $C H S-A$ gene $[24,36,37$, this study], suggest that a conserved feature in exon 2 of the CHS gene across plant species, e.g., the secondary structure of transcripts and/or termination of transcription, is a key element involved in the induction of CHS RNA degradation. We mapped highly abundant siRNAs on the secondary structure of $C H S-A$ RNA predicted by using $\mathrm{m}$-fold software [45]. Some of the highly abundant siRNAs were mapped within limited regions that formed an incomplete dsRNA structure comprising both a stretch of base-pairing and an unpaired loop structure (Additional file 3: Figure S3). Such a structure is reminiscent of the fact that the presence of bulges adjacent to the cleavage site is important for processing primary miRNAs [46]. It is tempting to speculate that such a "partially opened" structure is preferred by DCL or RDR6 orthologue(s) and leads to the production of abundant siRNAs.

\section{Potential triggers of cosuppression and naturally occurring RNA silencing of the CHS-A gene}

Among the cases of naturally occurring RNA silencing so far reported, a triggering mechanism has been suggested for only a few cases, all of which involve production of dsRNA either through read-through transcription of duplicated and rearranged genes $[25,47,48]$ or through convergent transcription of an overlapping gene pair [49]. The presence of an inverted repeat comprising CHS genes or gene segments is correlated with $\mathrm{CHS}$ RNA silencing in soybean, and loss of such structures suppresses its induction in spontaneous mutants $[28,29]$. In petunia, the mechanism(s) responsible for naturally occurring $C H S-A$ RNA silencing is not known, aside from the fact that the silencing occurs via RNA degradation that involves siRNA production [23]. A correlation between naturally occurring $C H S-A$ RNA silencing that results in the star-type or picotee-type flower color pattern and the presence of two tandemly linked $C H S$ - $A$ genes has been reported in petunia [24]. However, these two $C H S$ - $A$ genes are separated by a long sequence (almost $7 \mathrm{~kb}$ ), and a causative relationship between RNA silencing and the presence of the two copies of the $C H S$ - $A$ gene has not been presented.

For sense RNA-mediated silencing such as cosuppression in transgenic plants, a threshold sensing model, in which aberrant single-stranded RNA that accumulates 


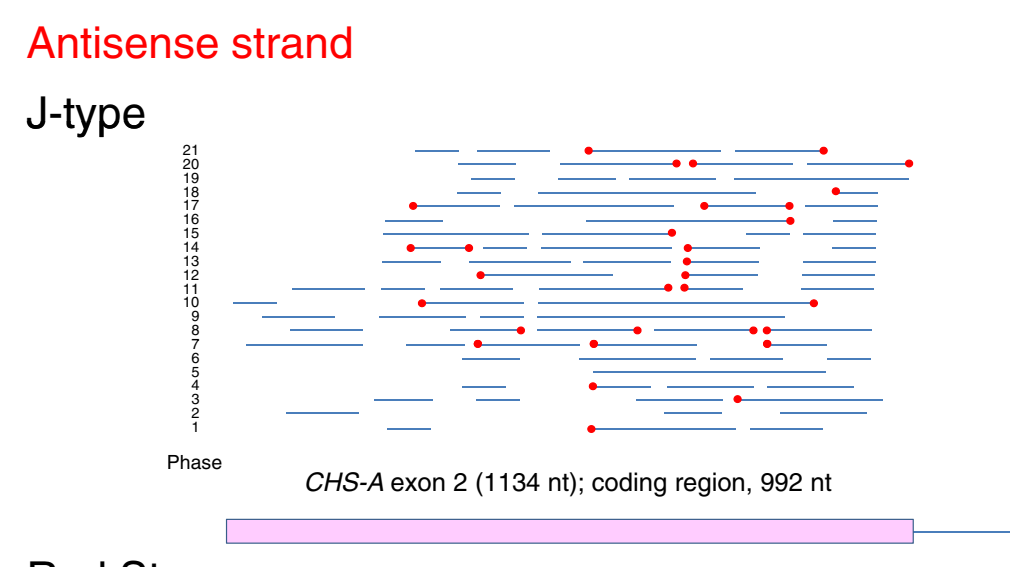

\section{Red Star}
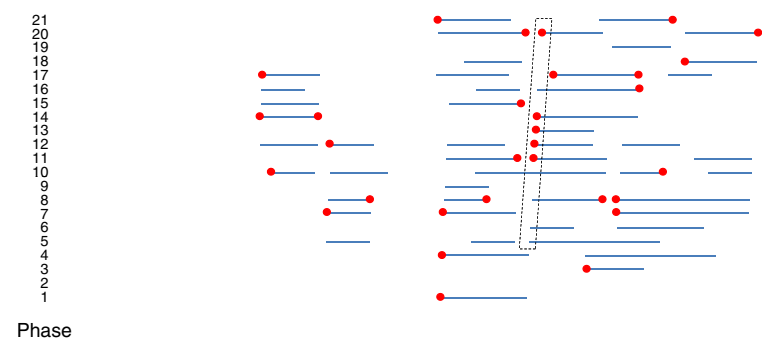

3 units of phased siRNAs (63 nt)

Figure 9 Phased siRNAs from antisense strand of CHS-A exon 2. The ends of phased siRNAs indicated by dotted lines are shown in detail in Figure 12. For other details, see legend to Figure 8.

beyond a critical level triggers its copying into dsRNA, has been suggested [50]. In fact, previous observations in $C H S-A$ cosuppressed petunias are consistent with this notion $[33,35]$. Meanwhile, De Paoli et al. reported the presence of two extra-abundant 21-nt siRNAs of antisense polarity of $C H S-A$, phy-siR1 and phy-siR2, in a $C H S-A$ cosuppressed petunia line and proposed that these siRNAs may trigger subsequent degradation of $C H S$ - $A$ transcripts [36]. On the other hand, we found that there are 21-nt siRNAs of both sense and antisense polarities that are more abundant than phy-siR1 and phy-siR2 (Figure 7; phy-siR1 and phy-siR2 are indicated by single and double asterisks, respectively). Moreover, no phased siRNAs whose end positions coincide with a cleavage in the middle of phy-siR1 or phy-siR2 were detected in this study (data not shown). These results, together with the presence of siRNAs in multiple phases, suggest that phy-siR1 and phy-siR2 are at least not the sole trigger for RNA degradation in different $C H S-A$ cosuppressed lines, although circumstantial evidence indicates that RNA cleavages with these siRNAs can induce phased siRNA production [36]. The reason for the difference between our data and that of De Paoli et al. is not known at present, but we speculate that a slight difference in the developmental stage of the flowers could affect the composition of the siRNA population. Such a possibility needs to be examined, but can be excluded in the comparison between the J-type and Red Star plants of this study because flower tissues of an identical developmental stage were used for our analysis. Our data suggest that the $C H S-A$ transcripts are cleaved at multiple, conserved positions in both J-type and Red Star plants. The siRNAs that guide these cleavages may include a potential trigger of RNA silencing. Whether a single cleavage of RNA can lead to extensive RNA degradation through RNA silencing pathways in these silencing systems is an issue to be addressed.

\section{The presence of siRNA at a low level in pigmented cells} We found that $C H S-A$ siRNA was present in pigmented portions in both J-type and Red Star plants at a low level. On the other hand, an extremely low level (only 2 reads) of CHS-A siRNA was detected in 16,651,540 total reads for line V26 (data not shown), a wild-type plant that produces completely purple flowers and was used to produce J-type plants through the introduction of the $C H S-A$ transgene. Therefore, the presence of CHS-A siRNAs in the pigmented petal tissues in J-type plants is 

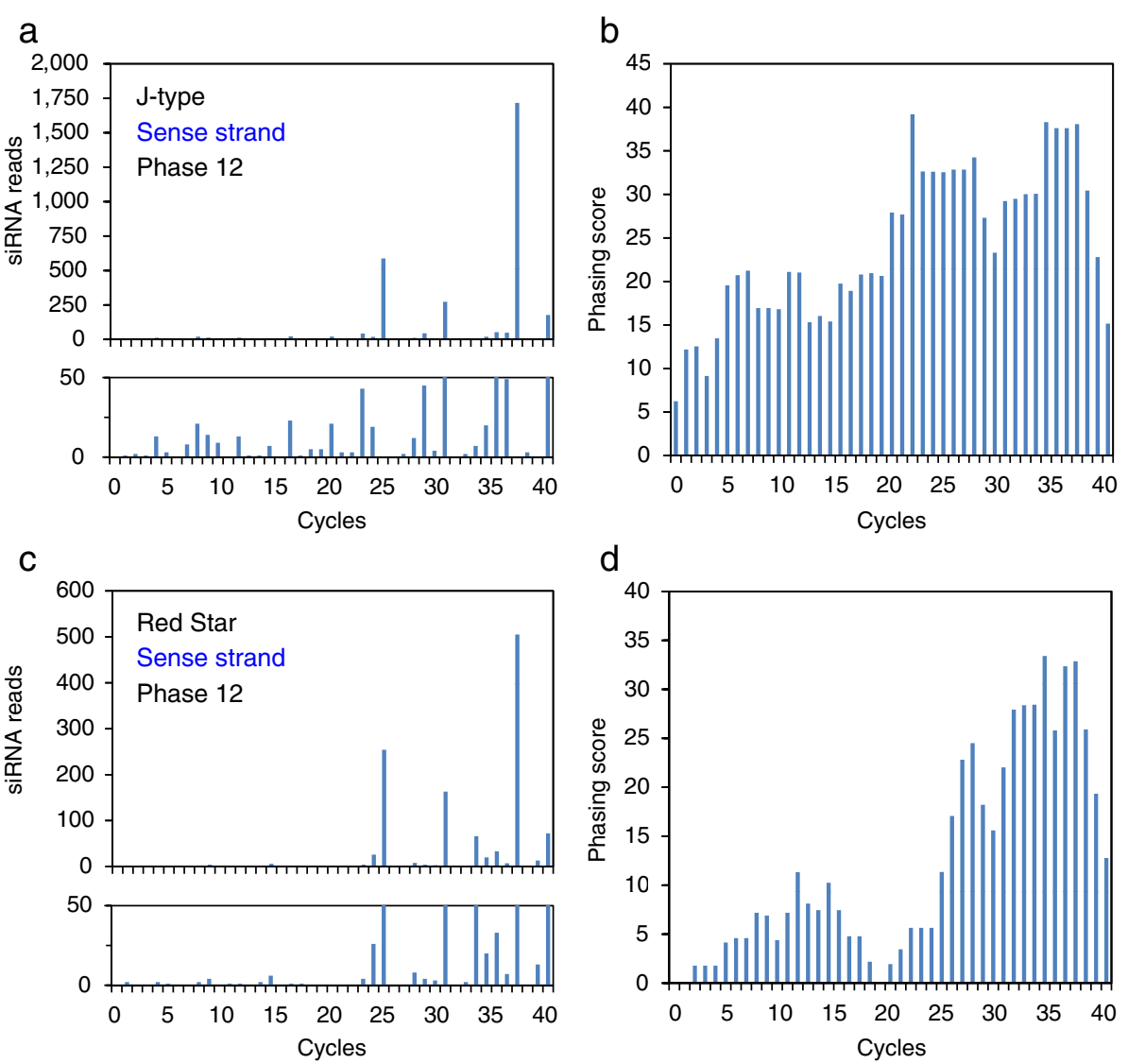

Figure 10 Abundance of 21-nt siRNAs and phasing scores of sense strand. Data of phase 12 of sense strand siRNAs, in which total number of contiguous siRNA units was highest in J-type (see Figure 8), are shown. $\mathbf{a}$, siRNA reads in J-type; $\mathbf{b}$, phasing score of the data in panel a; $\mathbf{c}$, siRNA reads in Red Star; d, phasing score of the data in panel c. In panels a and c, a close up of the graphs up to 50 reads are shown below. Cycle 1 corresponds to the phased siRNA mapped at the upstream end of contiguous units in J-type (Figure 8). Phasing scores are calculated according to Howell et al. (2007).

associated with cosuppression that occurred in other cells of the petal tissue.

A likely explanation for the presence of $C H S$ - $A$ siRNA in pigmented cells is that RNA is degraded at a low rate in the pigmented cells. Alternatively, the siRNAs may migrate from cells that underwent PTGS through plasmodesmata. In either case, these results raise a novel possibility that a threshold level of $C H S$ - $A$ siRNAs might be associated with extensive RNA degradation in addition to the previous idea that an aberrant CHS-A primary transcript level constitutes such a threshold level. It would not be surprising that, taking into account the observed commonality in siRNA profiles between these two silencing systems, they share a common sensing mechanism for trigger RNAs.

\section{Conclusions}

The present study revealed common features in siRNA production of the $C H S-A$ gene between cosuppression in transgenic plants and naturally occurring silencing in nontransgenic plants of petunia. In both silencing systems, 21-nt and 22-nt siRNAs were the first- and the second-most abundant size classes, respectively. $C H S-A$ siRNA production was confined to exon 2, indicating that $C H S$ - $A$ RNA is degraded through processes including cleavage and secondary siRNA production in this exon. Common siRNAs were detected in cosuppression and naturally occurring RNA silencing, whose ranks, according to the number of siRNAs in these plants, were correlated with each other. Highly abundant siRNAs were produced from multiple sites, many of which were common to the two silencing systems. Phased siRNAs were detected in multiple phases, and some of the ends of the regions that produced phased siRNAs were conserved. These results indicate mechanistic similarity between cosuppression and naturally occurring RNA silencing of the CHS-A gene, especially in the biosynthetic processes of siRNAs including cleavage of $C H S$ - $A$ transcripts and subsequent production of secondary siRNAs, which presumably depend on the 

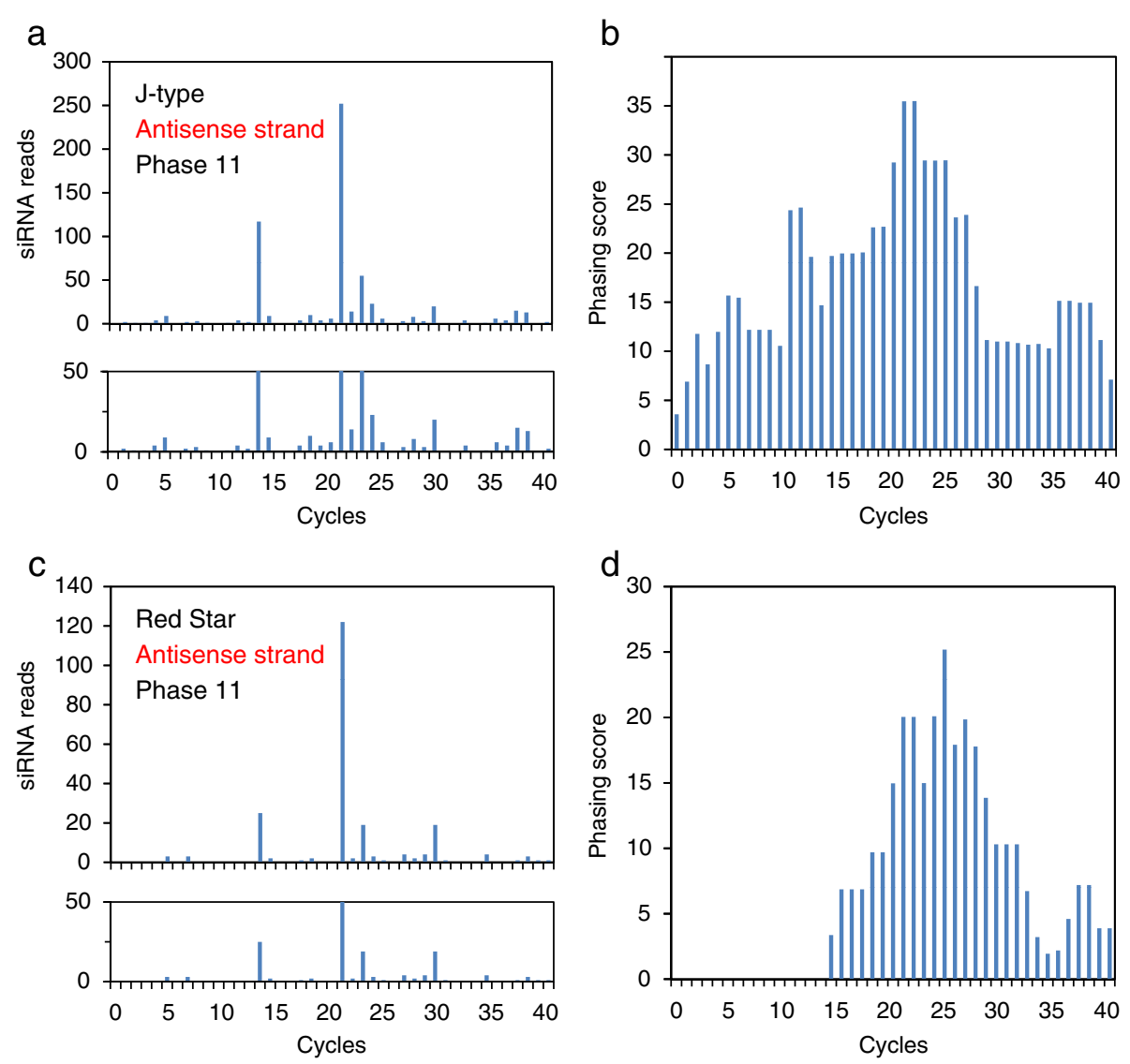

Figure 11 Abundance of 21-nt siRNAs and phasing scores of antisense strand. Data of phase 11 of antisense strand siRNAs, in which total number of contiguous siRNA units was highest in J-type (see Figure 9), are shown. Cycle 1 corresponds to the phased siRNA mapped at the upstream end of contiguous units in J-type (Figure 9). For other details, see legend to Figure 10.

nucleotide sequence and/or structural features of exon 2 RNA.

\section{Methods}

\section{Plant materials}

Petunia hybrida variety Red Star (Takii Seed Co., Japan) and a transgenic petunia line that produces junctiontype flowers (J-type) [33] were used for analyses. The transgenic line is a descendent of the CHS223 line $[19,51]$ and contains a single-copy $C H S-A$ transgene. The white and the pigmented petal tissues of these plants were analyzed separately. Petal tissues were used at the developmental stage when the mRNA level of the $C H S$ - $A$ gene is highest [52].

\section{Isolation of total RNA and RT-PCR}

Isolation of total RNA from flower tissues, cDNA synthesis, and RT-PCR were done as described previously [37]. The following primer pairs were used for the PCR: for the CHS-A gene, 4246 (5'-GGCGCGATCA TTATAGGTTC-3') and 5003 (5'-TTTGAGATCAG CCCAGGAAC-3'); for the $\alpha$-tubulin gene, tub $125 \mathrm{~F}$
(5' -CAACTATCAGCCACCAACTG-3') and tub 267R (5'-CACGCTTGGCATACATCAGA-3').

\section{Northern blot analysis of siRNA}

Low-molecular-weight RNA was isolated, and CHS-A siRNAs were detected by Northern blot analysis using a digoxigenin-labeled probe essentially as described by Goto et al. [53]. The following modifications were applied: RNA extraction buffer contained $100 \mathrm{mM}$ Tris- $\mathrm{HCl}$ (pH 8.8), $20 \mathrm{mM}$ EDTA, $200 \mathrm{mM} \mathrm{NaCl}$ and $4 \% \mathrm{~N}$-lauroyl sarcosine; an RNA probe specific for $\mathrm{CHS}$ $A$ antisense RNA was labeled by in vitro transcription of the plasmid carrying a $0.44-\mathrm{kb}$ region of the CHS-A gene [53] using DIG RNA labeling kit (Roche Applied Science, Basel, Switzerland) for use in hybridizations.

\section{Deep sequencing analysis of siRNA}

Low-molecular-weight RNA was extracted from the petal tissues of flower buds before the buds opened ( $\sim 4.5 \mathrm{~cm}$ long for J-type and $\sim 5.0 \mathrm{~cm}$ long for Red Star). Tissues were frozen with liquid nitrogen and extracted with RNA extraction buffer containing 
$10 \mathrm{mM}$ Tris- $\mathrm{HCl}$ (pH 7.5), $100 \mathrm{mM} \mathrm{NaCl}, 1 \mathrm{mM}$ EDTA, and $1 \%(\mathrm{w} / \mathrm{v})$ SDS. After extraction with phenol/chloroform, high-molecular-weight RNA was precipitated by mixing the aqueous phase with $1 / 3$ volume of $8 \mathrm{M} \mathrm{LiCl}$. After the solution was kept on ice overnight, the solution was centrifuged, and the nucleic acids in the supernatant were precipitated with ethanol. After centrifugation, the pellet was dissolved in water, and an equal amount of $20 \%$ polyethylene glycol $(\mathrm{MW}=8000)$ was added to the solution to separate high-molecular-weight nucleic acids. The solution was held on ice for $1 \mathrm{~h}$, then centrifuged, and low-molecular-weight RNA in the supernatant was precipitated with ethanol. After centrifugation, the pellet was dissolved in water and used for the following reactions. Low-molecular-weight RNA was ligated to $5^{\prime}$ - and $3^{\prime}$-RNA adapters, reverse transcribed, and amplified by PCR using a Small RNA Sample Prep Kit (Illumina, San Diego, CA, USA) according to the manufacturer's protocol except that we separated small RNAs by electrophoresis on a 3\% agarose gel instead of an acrylamide gel. Nucleotide sequence of the amplified cDNA was analyzed using an Illumina Genome Analyzer. The adapter sequence was trimmed from the raw short-read data, and the resulting short reads (15-45 nt) were mapped to the nucleotide sequence of the CHS-A gene region (EMBL/GenBank/DDBJ database accession X14591), allowing only perfect matches. Nucleotide positions in this study correspond to those on this sequence. The secondary structure of CHS-A sense and antisense RNAs was predicted by $\mathrm{m}$-fold software [45]. Correlation between the rank of the siRNA of J-type and Red Star plants was evaluated by Spearman's rank correlation coefficient. Phased siRNAs were detected by independently mapping siRNAs of the CHS- $A$ gene in 21 different phases. Calculation of phasing scores and assignment of scores to cycle position were done according to Howell et al. [54]. Nucleotide sequence data have been deposited in NCBI's Gene Expression Omnibus and are accessible through GEO Series accession number GSE42965.

\section{Additional files}

Additional file 1: Figure S1. Highly abundant 22-nt siRNAs in white portion of J-type and Red Star petals. The siRNAs of sense (a) and antisense (b) strands were ordered according to the number of reads in deep-sequencing analysis. J-w and R-W refer to the siRNAs detected in white portions of J-type and Red Star petals, respectively. The 10 most abundant siRNAs in J-w are colored, and the siRNAs of the same sequence in Red Star are colored the same. Note that most of the highly abundant siRNAs in J-type plants are also highly abundant in Red Star plants.

Additional file 2: Figure S2. RNA cleavage at various sites that initiate production of siRNA can be a feature of siRNA production common to cosuppression and naturally occurring RNA silencing of the CHS-A gene. siRNAs are produced from DCL cleavage of secondary-structured nascent CHS-A transcripts or dsRNAs produced by RDR6 orthologue(s) from the nascent transcripts. These siRNAs then cleave the CHS-A RNA at the target site with AGO, which triggers RDR6-mediated dsRNA production and subsequent DCL cleavage that produces phased siRNAs.

Additional file 3: Figure S3. Commonality of the siRNA hot spots between J-type and Red Star petals. Abundant siRNAs in the white tissues of J-type and Red Star petals are mapped on a secondary structure (a) and antisense (b) strands predicted by m-fold. Close ups of major hot spots are shown in windows, in which positions of nucleotides 
corresponding to siRNAs are marked by circles. Darker colors represent more total reads of siRNAs that contain the nucleotide. Mapped positions of siRNAs often overlapped, so that neighboring nucleotides had different colors. Nucleotide positions of abundant siRNAs mapped in each region are listed in the corresponding windows. Ranks of siRNA according to read number (see Figure 7) are in parentheses.

\section{Abbreviations}

AGO: Argonaute; CaMV: Cauliflower mosaic virus; CHS: Chalcone synthase; DCL: Dicer-like; dsRNA: Double-stranded RNA; IR: Inverted repeat; J-type: Junction type; miRNA: microRNA; NOS: Nopaline synthase; PTGS: Posttranscriptional gene silencing; RDR: RNA-dependent RNA polymerase; S-PTGS: Sense-PTGS; siRNA: Short interfering RNA; tasiRNA: Trans-acting siRNA; TGS: Transcriptional gene silencing.

\section{Competing interests}

The authors declare that they have no competing interests.

\section{Authors' contributions}

AK conceived and planned the study. MK did the experiments including RNA isolation, RT-PCR, Northern blot analysis, and preparation of small RNA CDNA library. HM, KY and RT carried out deep-sequencing of small RNAs. AT classified and mapped small RNA reads onto the gene sequence. MK and AK characterized small RNAs and drafted the manuscript. All authors read and approved the final manuscript.

\section{Acknowledgments}

We thank Mineo Senda, Akihito Fukudome, and Kenji Nakahara for helpful discussion and Neal Gutterson and Richard Jorgensen for transgenic petunia plants. The work was supported by Grants-in-Aid for Scientific Research from the Ministry of Education, Culture, Sports, Science and Technology of Japan.

\section{Author details}

${ }^{1}$ Research Faculty of Agriculture, Hokkaido University, Sapporo 060-8589, Japan. ${ }^{2}$ Gene Research Center, Shinshu University, Ueda 386-8567, Japan. ${ }^{3}$ Iwate Biotechnology Research Center, Kitakami 024-0003, Japan. ${ }^{4}$ Graduate School of Science and Technology, Hirosaki University, Hirosaki, Aomori 036-8561, Japan.

Received: 1 November 2012 Accepted: 22 January 2013 Published: 30 January 2013

\section{References}

1. Baulcombe D: RNA silencing in plants. Nature 2004, 431:356-363.

2. Carmell MA, Hannon GJ: RNase III enzymes and the initiation of gene silencing. Nat Struct Mol Biol 2004, 11:214-218.

3. Fusaro AF, Matthew L, Smith NA, Curtin SJ, Dedic-Hagan J, Ellacott GA, Watson JM, Wang MB, Brosnan C, Carroll BJ, Waterhouse PM: RNA interference-inducing hairpin RNAs in plants act through the viral defence pathway. EMBO Rep 2006, 7:1168-1175.

4. Brodersen $\mathrm{P}$, Voinnet $\mathrm{O}$ : The diversity of RNA silencing pathways in plants. Trends Genet 2006, 22:268-280

5. Vaucheret H: Plant ARGONAUTES. Trends Plant Sci 2008, 13:350-358,

6. Mette MF, Aufsatz W, van der Winden J, Matzke MA, Matzke AJ: Transcriptional silencing and promoter methylation triggered by doublestranded RNA. EMBO J 2000, 19:5194-5201.

7. Jones L, Ratcliff F, Baulcombe DC: RNA-directed transcriptional gene silencing in plants can be inherited independently of the RNA trigger and requires Met1 for maintenance. Curr Biol 2001, 11:747-757.

8. Sijen T, Vijn I, Rebocho A, van Blokland R, Roelofs D, Mol JN, Kooter JM: Transcriptional and posttranscriptional gene silencing are mechanistically related. Curr Biol 2001, 11:436-440.

9. Mallory AC, Vaucheret $\mathrm{H}$ : Functions of microRNAs and related small RNAs in plants. Nat Genet 2006, 38:S31-S36.

10. Peragine A, Yoshikawa M, Wu G, Albrecht HL, Poethig RS: SGS3 and SGS2/ SDE1/RDR6 are required for juvenile development and the production of trans-acting siRNAs in Arabidopsis. Genes Dev 2004, 18:2368-2379.

11. Vazquez F, Vaucheret H, Rajagopalan R, Lepers C, Gasciolli V, Mallory AC, Hilbert JL, Bartel DP, Crété P: Endogenous trans-acting siRNAs regulate the accumulation of Arabidopsis mRNAs. Mol Cell 2004, 16:69-79
12. Allen E, Xie Z, Gustafson AM, Carrington JC: microRNA-directed phasing during trans-acting siRNA biogenesis in plants. Cell 2005, 121:207-221.

13. Chen HM, Chen LT, Patel K, Li YH, Baulcombe DC, Wu SH: 22-nucleotide RNAs trigger secondary siRNA biogenesis in plants. Proc Natl Acad Sci U S A 2010, 107:15269-15274.

14. Cuperus JT, Carbonell A, Fahlgren N, Garcia-Ruiz H, Burke RT, Takeda A, Sullivan CM, Gilbert SD, Montgomery TA, Carrington JC: Unique functionality of 22-nt miRNAs in triggering RDR6-dependent siRNA biogenesis from target transcripts in Arabidopsis. Nat Struct Mol Biol 2010, 17:997-1003.

15. Manavella PA, Koenig D, Weigel D: Plant secondary siRNA production determined by microRNA-duplex structure. Proc Natl Acad Sci U S A 2012, 109:2461-2466.

16. Napoli C, Lemieux C, Jorgensen R: Introduction of a chimeric chalcone synthase gene into petunia results in reversible co-suppression of homologous genes in trans. Plant Cell 1990, 2:279-289.

17. van der Krol AR, Mur LA, Beld M, Mol JN, Stuitje AR: Flavonoid genes in petunia: addition of a limited number of gene copies may lead to a suppression of gene expression. Plant Cell 1990, 2:291-299.

18. Jorgensen RA: Cosuppression, flower color patterns, and metastable gene expression Status. Science 1995, 268:686-691.

19. Jorgensen RA, Cluster PD, English J, Que Q, Napoli CA: Chalcone synthase cosuppression phenotypes in petunia flowers: comparison of sense vs. antisense constructs and single-copy vs. complex T-DNA sequences. Plant Mol Biol 1996, 31:957-973.

20. Kanazawa A: RNA silencing manifested as visibly altered phenotypes in plants. Plant Biotechnol 2008, 25:423-435.

21. Kanazawa A, Inaba J, Shimura H, Otagaki S, Tsukahara S, Matsuzawa A, Kim BM, Goto K, Masuta C: Virus-mediated efficient induction of epigenetic modifications of endogenous genes with phenotypic changes in plants. Plant J 2011, 65:156-168.

22. Kanazawa A, Inaba J, Kasai M, Shimura H, Masuta C: RNA-mediated epigenetic modifications of an endogenous gene targeted by a viral vector: a potent gene silencing system to produce a plant that does not carry a transgene but has altered traits. Plant Signal Behav 2011, 6:1090-1093.

23. Koseki M, Goto K, Masuta C, Kanazawa A: The star-type color pattern in Petunia hybrida 'Red Star' flowers is induced by sequence-specific degradation of chalcone synthase RNA. Plant Cell Physiol 2005, 46:1879-1883.

24. Morita Y, Saito R, Ban Y, Tanikawa N, Kuchitsu K, Ando T, Yoshikawa M, Habu Y, Ozeki Y, Nakayama M: Tandemly arranged chalcone synthase A genes contribute to the spatially regulated expression of siRNA and the natural bicolor floral phenotype in Petunia hybrida. Plant J 2012, 70:739-749.

25. Kusaba M, Miyahara K, lida S, Fukuoka H, Takano T, Sassa H, Nishimura M, Nishio T: Low glutelin content1: a dominant mutation that suppresses the glutelin multigene family via RNA silencing in rice. Plant Cell 2003, 15:1455-1467.

26. Senda M, Masuta C, Ohnishi S, Goto K, Kasai A, Sano T, Hong JS, MacFarlane S: Patterning of virus-infected Glycine max seed coat is associated with suppression of endogenous silencing of chalcone synthase genes. Plant Cell 2004, 16:807-818.

27. Tuteja JH, Clough SJ, Chan WC, Vodkin LO: Tissue-specific gene silencing mediated by a naturally occurring chalcone synthase gene cluster in Glycine max. Plant Cell 2004, 16:819-835.

28. Tuteja JH, Zabala G, Varala K, Hudson M, Vodkin LO: Endogenous, tissuespecific short interfering RNAs silence the chalcone synthase gene family in Glycine max seed coats. Plant Cell 2009, 21:3063-3077.

29. Senda M, Kurauchi T, Kasai A, Ohnishi S: Suppressive mechanism of seed coat pigmentation in yellow soybean. Breed Sci 2012, 61:523-530.

30. Della Vedova CB, Lorbiecke R, Kirsch H, Schulte MB, Scheets K, Borchert LM Scheffler BE, Wienand U, Cone KC, Birchler JA: The dominant inhibitory chalcone synthase allele C2-Idf (Inhibitor diffuse) from Zea mays (L.) acts via an endogenous RNA silencing mechanism. Genetics 2005, 170:1989-2002.

31. Ohno S, Hosokawa M, Kojima M, Kitamura Y, Hoshino A, Tatsuzawa F, Doi M, Yazawa S: Simultaneous post-transcriptional gene silencing of two different chalcone synthase genes resulting in pure white flowers in the octoploid dahlia. Planta 2011, 234:945-958.

32. Wang MB, Metzlaff M: RNA silencing and antiviral defense in plants. Curr Opin Plant Biol 2005, 8:216-222.

33. Kanazawa A, O'Dell M, Hellens RP: Epigenetic inactivation of chalcone synthase- $A$ transgene transcription in petunia leads to a reversion of the 
post-transcriptional gene silencing phenotype. Plant Cell Physiol 2007, 48:638-647.

34. Smith HA, Swaney SL, Parks TD, Wernsman EA, Dougherty WG: Transgenic plant virus resistance mediated by untranslatable sense RNAs: expression, regulation, and fate of nonessential RNAs. Plant Cell 1994, 6:1441-1453.

35. Que Q, Wang HY, English JJ, Jorgensen RA: The frequency and degree of cosuppression by sense chalcone synthase transgenes are dependent on transgene promoter strength and are reduced by premature nonsense codons in the transgene coding sequence. Plant Cell 1997, 9:1357-1368.

36. De Paoli E, Dorantes-Acosta A, Zhai J, Accerbi M, Jeong DH, Park S, Meyers BC, Jorgensen RA, Green PJ: Distinct extremely abundant siRNAs associated with cosuppression in petunia. RNA 2009, 15:1965-1970.

37. Kasai M, Koseki M, Goto K, Masuta C, Ishii S, Hellens RP, Taneda A, Kanazawa A: Coincident sequence-specific RNA degradation of linked transgenes in the plant genome. Plant Mol Biol 2012, 78:259-273.

38. Metzlaff M, O'Dell M, Cluster PD, Flavell RB: RNA-mediated RNA degradation and chalcone synthase A silencing in petunia. Cell 1997, 88:845-854.

39. Vermeersch L, De Winne N, Depicker A: Introns reduce transitivity proportionally to their length, suggesting that silencing spreads along the pre-mRNA. Plant J 2010, 64:392-401.

40. Christie M, Croft LJ, Carroll BJ: Intron splicing suppresses RNA silencing in Arabidopsis. Plant J 2011, 68:159-167.

41. Wahl MC, Will CL, Lührmann R: The spliceosome: design principles of a dynamic RNP machine. Cell 2009, 136:701-718.

42. Le Hir H, Moore MJ, Maquat LE: Pre-mRNA splicing alters mRNP composition: evidence for stable association of proteins at exon-exon junctions. Genes Dev 2000, 14:1098-1108.

43. Axtell MJ, Jan C, Rajagopalan R, Bartel DP: A two-hit trigger for siRNA biogenesis in plants. Cell 2006, 127:565-577.

44. Kurauchi T, Matsumoto T, Taneda A, Sano T, Senda M: Endogenous short interfering RNAs of chalcone synthase genes associated with inhibition of seed coat pigmentation in soybean. Breed Sci 2009, 59:419-426.

45. Zuker M: Mfold web server for nucleic acid folding and hybridization prediction. Nucleic Acids Res 2003, 31:3406-3415.

46. Song L, Axtell MJ, Fedoroff NV: RNA secondary structural determinants of miRNA precursor processing in Arabidopsis. Curr Biol 2010, 20:37-41.

47. Melquist $\mathrm{S}$, Bender J: Transcription from an upstream promoter controls methylation signaling from an inverted repeat of endogenous genes in Arabidopsis. Genes Dev 2003, 17:2036-2047.

48. Kasai A, Kasai K, Yumoto S, Senda M: Structural features of GmIRCHS, candidate of the I gene inhibiting seed coat pigmentation in soybean: implications for inducing endogenous RNA silencing of chalcone synthase genes. Plant Mol Biol 2007, 64:467-479.

49. Borsani O, Zhu J, Verslues PE, Sunkar R, Zhu JK: Endogenous siRNAs derived from a pair of natural cis-antisense transcripts regulate salt tolerance in Arabidopsis. Cell 2005, 123:1279-1291.

50. Tomari Y, Zamore PD: Perspective: machines for RNAi. Genes Dev 2005, 19:517-529.

51. Cluster PD, O'Dell M, Metzlaff M, Flavell RB: Details of T-DNA structural organization from a transgenic Petunia population exhibiting cosuppression. Plant Mol Biol 1996, 32:1197-1203.

52. Koes R, Spelt C, Mol J: The chalcone synthase multigene family of Petunia hybrida (V30): differential, light-regulated expression during flower development and UV-light induction. Plant Mol Biol 1989, 12:213-225.

53. Goto K, Kanazawa A, Kusaba M, Masuta C: A simple and rapid method to detect plant siRNAs using nonradioactive probes. Plant Mor Biol Rep 2003, 21:51-58.

54. Howell MD, Fahlgren N, Chapman EJ, Cumbie JS, Sullivan CM, Givan SA, Kasschau KD, Carrington JC: Genome-wide analysis of the RNADEPENDENT RNA POLYMERASE6/DICER-LIKE4 pathway in Arabidopsis reveals dependency on miRNA- and tasiRNA-directed targeting. Plant Cell 2007, 19:926-942.

doi:10.1186/1471-2164-14-63

Cite this article as: Kasai et al.: Deep sequencing uncovers commonality in small RNA profiles between transgene-induced and naturally occurring RNA silencing of chalcone synthase-A gene in petunia. BMC Genomics 2013 14:63.

\section{Submit your next manuscript to BioMed Central and take full advantage of:}

- Convenient online submission

- Thorough peer review

- No space constraints or color figure charges

- Immediate publication on acceptance

- Inclusion in PubMed, CAS, Scopus and Google Scholar

- Research which is freely available for redistribution 